\title{
Mechanical Properties of GF/pCBT Composites and Their Fusion-Bonded Joints: Influence of Process Parameters
}

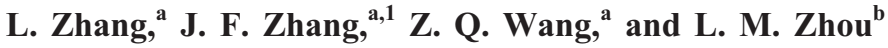

a Smart Structures and Composites Laboratory, Department of Aerospace, Harbin Engineering University, Harbin, China

${ }^{b}$ Department of Mechanical Engineering, The Hong Kong Polytechnic University, Hong Kong, China

1 jfzhang@hrbeu.edu.cn

High melting viscosity of thermoplastic composites gives no way of using substantial volume fractions of reinforcing agents. This problem can be solved by in-situ polymerization of an extremely low-viscosity cyclic butylene terephthalate (CBT) resin. Continuous glass fiber-reinforced poly(cyclic butylene terephthalate) (GF/pCBT) composites with high fiber fractions were manufactured, and the mechanical properties as a function of the catalyst mass fraction and fiber filling ratio were studied. The longitudinal tensile strength of the composites was enhanced by increasing the fiber volume fraction, and the influence of the fiber fraction on the bending strength of high fiber filling-ratio composites was evaluated. Furthermore, the mechanical properties and failure modes of GF/pCBT fusion-bonded joints with different number of bonding areas of different lengths were investigated. It was found that high-strength composite materials can be obtained, which are applicable for fusion-bonded structures..

Keywords: poly(cyclic butylene terephthalate), catalyst ratio, fiber volume fraction, fusion bonding, connection layers, connection length, mechanical properties.

Introduction. Thermoplastic composites are in the focus of numerous studies due to their attractive features, such as improved toughness, impact resistance, recoverability, short manufacturing cycle, and low production cost. However, application of continuous fiber-reinforced thermoplastic composites for production of primary load-carrying structures is problematic, since a high melting viscosity $(100 \sim 10000 \mathrm{~Pa} \cdot \mathrm{s})$ of their matrix hampers the impregnation and wetting of fibers $[1,2]$. In this respect, a new thermoplastic composite - cyclic butylene terephthalate (CBT) - is considered to be a promising material to solve this problem, particularly for major force-bearing components. The melting viscosity of CBT resin can reach extremely low values $\left(0.017 \mathrm{~Pa} \cdot \mathrm{s}\right.$ at $\left.190^{\circ} \mathrm{C}\right)$, because of its small molecular weight and macrocyclic molecular structure to simplify impregnation and wetting [3,4]. These unique characteristics provides possibilities to manufacture fiber-reinforced thermoplastic composites with the perfect impregnation of fibers, as well as to apply thermoset production methods, such as resin transfer molding (RTM) and vacuum assisted resin transfer molding (VARTM) [5]. It is crucial that CBT can be polymerized into poly(cyclic butylene terephthalate) (pCBT) in the catalyst presence via entropically driven ring-opening polymerization with an adjustable reaction time. Moreover, there are no volatile organic compounds and heat released during the reaction [6, 7].

The influence of the preparation temperature on the mechanical properties of GF/pCBT composites was investigated by Parton [8], who used $0.45 \%$ catalyst to manufacture the composites by RTM. On this basis, a simplified VARTM combined with a hot-pressing technology was applied to prepare $\mathrm{GF} / \mathrm{pCBT}$ composites with high fiber volume fraction in this work. In addition, the effects of catalyst mass fraction $(0.3,0.4,0.5,0.6$, and $0.7 \%)$ and fiber volume fraction $(50,60,65,70$, and $75 \%)$ on the mechanical properties were 
investigated. Then, the fusion bonding joints with different number of the connection layers $(1,2$, and 3$)$ and connection length $(50 \sim 110 \mathrm{~mm})$ were manufactured, in order to determine the mechanical properties and failure modes.

\section{Experiment.}

1.1. Materials. The CBT oligomers (CBT100) used in this study were produced by the Cyclics Corporation, and were subjected to dryinig in $110^{\circ} \mathrm{C}$ vacuum drying oven for more than $10 \mathrm{~h}$ prior to processing, so as to prevent the water effect on the polymerization reaction, as well as the hydrolysis of the polymer [9]. The polymerization catalyst butyl tin chloride dihydroxide (PC4101) was produced by the Canton Yuansu Chemical Company. The isopropanol was supplied by Tianjin Dekai Chemicals Business as the dissolving solution of catalyst. The reinforcement was unidirectional glass fabric material, which was delivered by Yixing Fuxing Glass Fiber Corporation, with an areal density of $300 \mathrm{~g} / \mathrm{m}^{2}$.

1.2. Preparation of $G F / p C B T$ Composite Laminates. When the catalyst was added, the CBT polymerization reaction occurred immediately, and the viscosity of CBT melt increased quickly, which made the process of fiber-impregnating quite difficult. In this study, the catalyst and CBT resin were sequestered, in order to solve this problem. The specific methods were used as follows: the catalyst was poured into isopropanol solution, stirred with magnetic stirrer at $75^{\circ} \mathrm{C}$ for $10 \mathrm{~min}$, the solution was brushed evenly onto the glass fabric after catalyst dissolved completely, and was oven-dried. This process provided the catalyst adhesion to the glass fabric. In situ polymerization on the surface of the glass fabric occured, when the glass fabric was impregnated resin.

VARTM and hot-pressing technology were combined to prepare GF/pCBT composite with a high fiber volume fraction, the particular technology being as follows: the cavity of the mold had a height of $2.4 \mathrm{~mm}$ and a length of $300 \mathrm{~mm}$. The fiber fabric with the catalyst was put into the mold, fluoroelastomer sealing strip was embedded somewhat higher than the thickness of the fiber fabric between the moulds. The heating temperature of hot press, injection tube and the oil bath tank was $230^{\circ} \mathrm{C}$. The ball valve was opened after the mold vacuumed, then resin was injected into the mould, and $5 \mathrm{MPa}$ pressure was exerted on the mould with hot press, it was held at $230^{\circ} \mathrm{C}$ for $20 \mathrm{~min}$, then cooled to $190^{\circ} \mathrm{C}$, and kept for $10 \mathrm{~min}$. After cooling to room temperature, the mold was taken out of the press and opened.

1.3. Preparation of Fusion-Bonded Joints. Composite laminates with grooves were manufactured as follows. The grooves were formed by the use of steel plates with a demoulding cloth instead of fibers upon completion of the preparation. Because the polymerized CBT resin melting point was $225^{\circ} \mathrm{C}$, the joint had to be heated to $235^{\circ} \mathrm{C}$, so that the two laminates with grooves could be connected together, in order to prepare the fusion-bonded joints of $\mathrm{GF} / \mathrm{pCBT}$ composites.

\section{Results and Discussion.}

2.1. Effects of the Catalyst Mass Fraction. To examine the effects of the catalyst rate on the mechanical properties of GF/pCBT composites, specimens with different catalyst mass fraction $(0.3,0.4,0.5,0.6$, and $0.7 \%)$ were manufactured by the above method. All composites were prepared using a longitudinal glass fiber cloth with a high volume filling ratio in fibers of $65 \%$. The tensile and flexural tests were performed using an Instron 4505 universal testing machine at room temperature in accordance with ASTM-D638 and ASTM-D790, respectively [10, 11].

The tensile and flexural strength values of the GF/pCBT composites are found to be increasing with the catalyst fraction values, when the latter are in the range of $0.3 \sim 0.6 \%$. However, the tensile/flexural strength values exhibit saturation and slow decrease with the catalyst mass fraction, when the latter increases to $0.6 \%$. This can be attributed to slow ring-opening polymerization of $\mathrm{CBT}$ and to the fact that the reaction occurs simultaneously with crystallization for lower catalyst fraction values [12], so that crystallization of pCBT appears to be incomplete. The crystalline content of $\mathrm{pCBT}$ rises with the catalyst ratio, which 
improves the mechanical properties of GF/pCBT composites. When the catalyst fraction exceeds $0.6 \%$, further increase in the catalyst ratio is not sufficient for accelerating the ring-opening reaction of $\mathrm{CBT}$, and increasing the crystalline content of $\mathrm{pCBT}$. The redundant catalyst turns into an impurity in the resin and, therefore, the mechanical properties decrease slightly [13]. Consequently, the mechanical properties of GF/pCBT composites are closely related to the catalyst volume fraction, while its appropriate value is about $0.6 \%$.

2.2. Effects of the Fiber Volume Fraction. Different $0^{\circ}$ unidirectional GF/pCBT composite specimens were produced with different (high) volume fractions $(50,60,65,70$, and $75 \%)$ and the same catalyst mass fraction $(0.6 \%)$ for investigating their tensile and flexural mechanical properties. The tests were carried out in comliance with the above standards using. five specimens of each fiber content $(50,60,65,70$, and $75 \%)$. The obtained results are shown in Fig. 1.

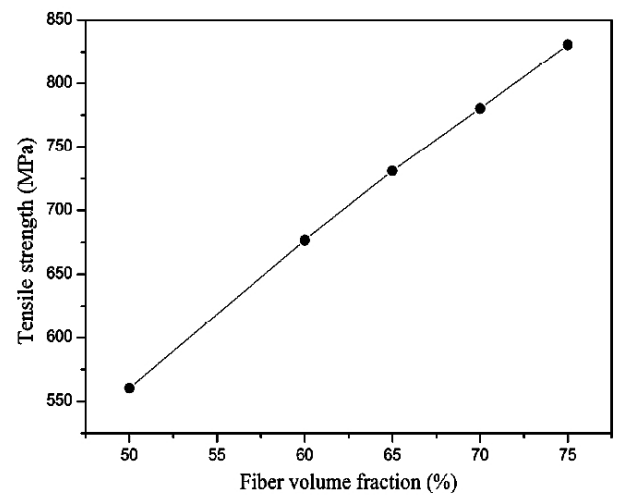

a

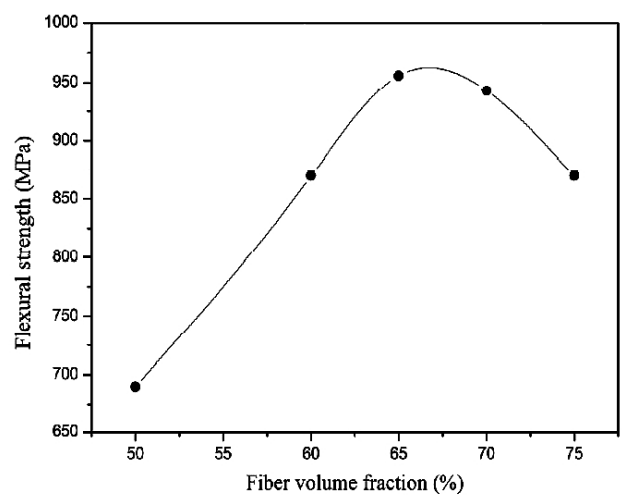

b

Fig. 1 Mechanical properties of GF/pCBT composites with different fiber content: tensile (a) and flexural (b) strengths of GF/pCBT composites.

Figure 1a shows that the longitudinal tensile strength $\sigma_{t}$ enhances with the increase of the fibers volume fraction $V_{f}$. This is obvious that the tensile strength of the glass fibers is larger than that of pCBT resin. Moreover, the evolution of $\sigma_{t}$ with $V_{f}$ is linear. The relation between them is as follows:

$$
\sigma_{t}=10.8 V_{f}+24.6
$$

where $\sigma_{t}$ values are given in $\mathrm{GPa}$ and $V_{f}$ in $\%$.

The variation of the longitudinal tensile strength with the volume fraction was revealed in numerous studies, while the relation between the mechanical properties of the fiber-reinforced composite and the fiber filling ratio was introduced in [14]. In case of a perfect cohesion between fibers and matrix, the relation between the tensile strength $\sigma_{t}$ and the fiber volume fraction $V_{f}$ can be reduced to Eq. (2):

$$
\sigma_{t}=\sigma_{f} V_{f}+\sigma_{m}^{*}\left(1-V_{f}\right)=\left(\sigma_{f}-\sigma_{m}^{*}\right) V_{f}+\sigma_{m}^{*},
$$

where $\sigma_{f}$ is the fiber tensile strength and $\sigma_{m}^{*}$ is the matrix stress, when the matrix strain is equal to the fiber fracture strain [15].

Substituting the exoerimental data on $\sigma_{f}$ and $\sigma_{m}^{*}$ into Eq. (2) yields the following relation between the fiber-reinforced composite longitudinal tensile strength and the fiber volume fraction $V_{f}$ : 


$$
\sigma_{t}=11.3 V_{f}+23.8
$$

which is similar to to the empirical formula in Eq. (1).

Figure $1 \mathrm{~b}$ depicts the flexural strength of the studied composites. In view of a relatively low fiber volume fraction, its increase improves the flexural strength. When the glass fiber volume fraction is $65 \%$, the flexural strength of GF/pCBT composite attains 956.2 $\mathrm{MPa}$ and then drops down with the further increase in the glass fiber fraction.

The above phenomenon can be attributed to the fact that the principal mechanism of energy absorption is degumming in the GF/pCBT composite fracture process. Therefore, for evenly distributed fibers a higher fiber content implies that the higher energy of interface failure is absorbed, which, in turn, inhibits the crack extension in the matrix and improves the composite material strength. The failure modes of composites are: (1) compression failure of the matrix near the surface and (2) tensile failure of the matrix near the bottom. However, when the fiber content is too high, fibers (as a result of the pruduction limitations) cannot effectively act as the enhancement. Instead, the fiber accumulation zone turns into a new weak link of composites and becomes susceptible to damage under stress [16]. As a result, the strength of composites deteriorates with an increase in the fiber content. In this case, the matrix failure and fiber delamination failure occur simultaneously. The fracture pattern for the composite under study is shown in Fig. 2.

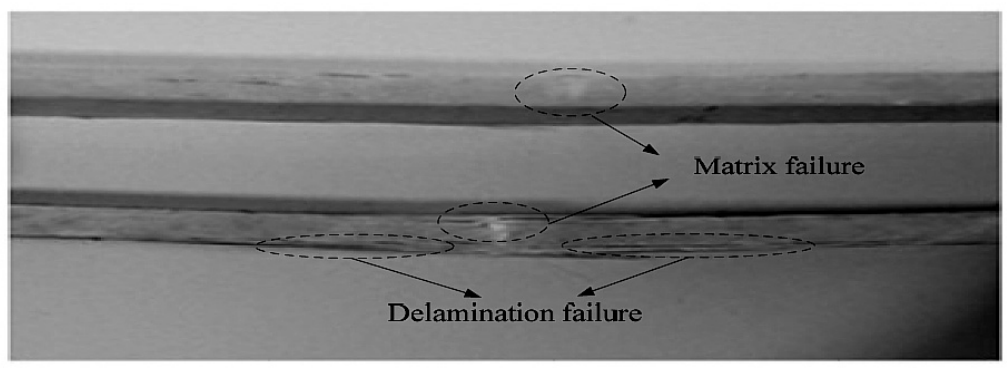

Fig. 2. Failure model of GF/pCBT composite specimens.

2.3. Effects of the Connection Layers. Three types of fusion-bonded joint specimens with different number of the connection layers $(1,2$, and 3$)$ were produced to study their mechanical properties. The connection schemes and geometries of joints are shown in Fig. 3. The ply sequence of composite laminates is $[0]_{6 S}$, and a single layer thickness is $0.2 \mathrm{~mm}$. The consolidated plates were cut to form $15 \mathrm{~mm}$-wide specimens. The tensile strength tests were conducted according to the above standards. Figure 4 shows examples of the load-displacement curves constructed in this study. The increased number of contact layers provides the tensile strength enhancement. As compared with joint I, the load-carrying capacities of joint II and joint III are improved by 35.5 and $85.9 \%$, respectively. Accordingly, when the number of the connection layers is in the range of $1 \sim 3$, the tensile properties can be enhanced with the number of connection layers. The mechanical properties of joint III are the maximal, which can be attributed to the main factor accelerating the expansion of interface crack due to the warping of composites in the joint area of joint I and joint II. However, the influence of warping on the strength of joint III is relatively small. The failure mode was identified in the specimens after their tensile testing. For all three types of the bonded regions, the main failure mode is the interface delamination.

2.4. Effects of the Connection Length. The joints with different connection length were manufactured to study the tensile behavior of fusion-bonded joints with the matrix of pCBT and glass fiber reinforcement. The connection length of type III joint was a single variable, since the strength of joint III is obviously higher than that of joint I. 
L. Zhang, J. F. Zhang, Z. Q. Wang, and L. M. Zhou

$\mathrm{T}$ a $\mathrm{b} 1 \mathrm{e} 1$

Critical Loads and Failure Modes of Joints with Different Connection Length Values

\begin{tabular}{|c|c|c|}
\hline Connection length $(\mathrm{mm})$ & Failure loads $(\mathrm{N})$ & Failure modes \\
\hline 50 & $9137.2 \pm 186.3$ & $\mathrm{~A}$ \\
\hline 60 & $12263.6 \pm 237.1$ & $\mathrm{~A}$ \\
\hline 70 & $14219.9 \pm 195.8$ & $\mathrm{~A}$ \\
\hline 80 & $14682.2 \pm 263.2$ & $\mathrm{~A}$ \\
\hline 90 & $15123.3 \pm 323.4$ & $\mathrm{~A}+\mathrm{B}$ \\
\hline 100 & $15133.2 \pm 281.1$ & $\mathrm{~B}$ \\
\hline 110 & $15119.6 \pm 361.5$ & $\mathrm{~B}$ \\
\hline
\end{tabular}

Note. A: delamination failure of interface layers; B: fiber tension failure of composites.

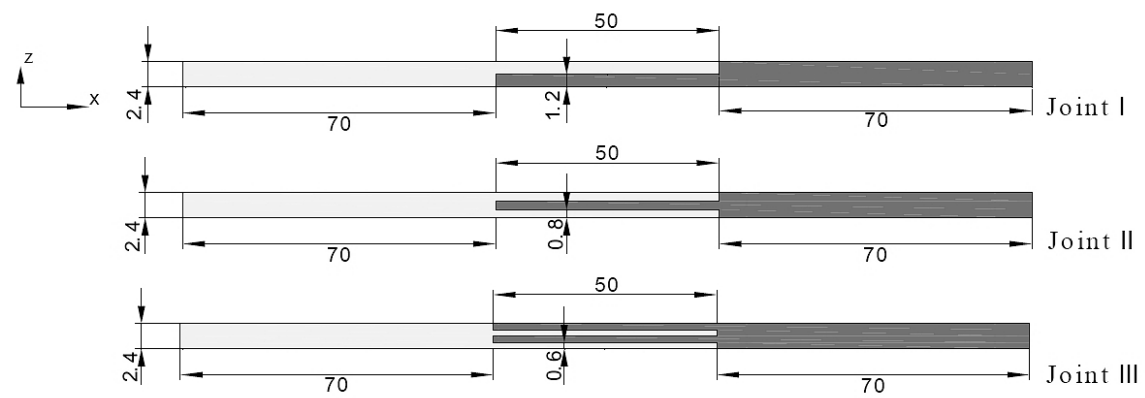

Fig. 3. Connection schemes and geometry of joints.

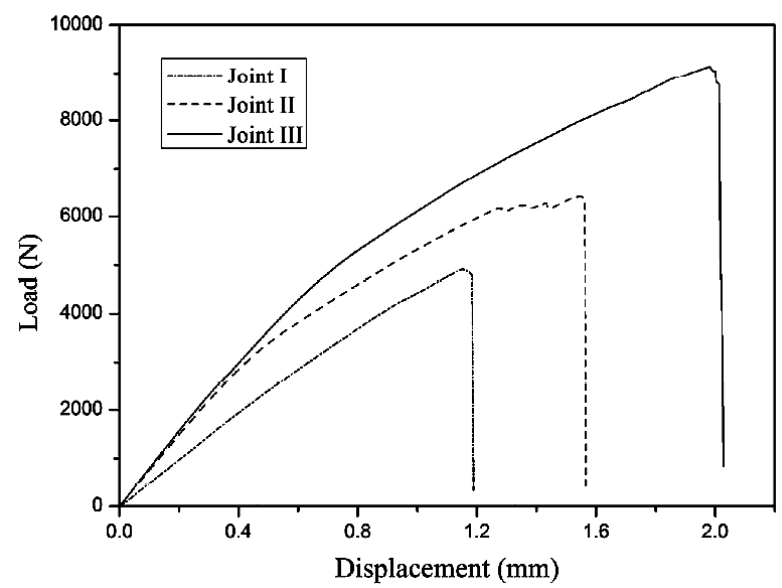

Fig. 4. Tensile behavior of fusion-bondine joints with different number of connection layers.

Table 1 shows the critical loads and failure modes of joints with different connection length values. The tensile properties of joints are improved with an increase in the connection length. However, the mechanical properties manifest a saturation at a $90 \mathrm{~mm}$ connection length, whereasthe interface delamination failure and fiber failure occur simultaneously. The tensile strength of glass fiber on the two ends of the joint is nearly equal to the shear strength of pCBT resin in the interface layers. With further increase in the connection length, the fiber failure becomes the main failure mode, while the load-carrying capacity exhibits no futher improvement. 
Conclusions. GF/pCBT composites with high fiber volume fraction via in-situ polymerization of CBT have been prepared by combining VARTM and a hot-pressing technology. The produced material is a laminate composed of unidirectional glass fiber plies. The optimal mechanical properties of GF/pCBT composites are observed, when the catalyst fraction is $0.6 \%$. The longitudinal tensile strength of composites increases with the rise of fiber content, and their evolution is linear. However, the maximal flexural strength of $0^{\circ}$ unidirectional composites was attained, when the volume percentage of glass fiber reached $65 \%$, and then decreased with fiber fraction. The analysis of this phenomenon implies that the agglomeration occurs at higher glass fiber filling ratio leading to an incompatibility between the fiber and the matrix.

In addition, several joints with a similar matrix reinforced with a different number of the connection layers $(1,2$, and 3$)$ and various connection lengths $(50 \sim 110 \mathrm{~mm})$ were designed and tested under tensile loading conditions. The respective mechanical properties and failure modes of the fusion-bonded joints were obtained. The tensile properties of joints attain relatively stable values at certain connection lengths and manifest their further saturation.

Finally, based on the performed analysis of the process parameters, all the experimental data on the mechanical behavior of the GF/pCBT composites and their fusion-bonded joints can be used to validate the optimal manufacturing technology of composite materials.

Acknowledgments. This work was supported by the Hong Kong Polytechnic University Research Grant (G-YF68 and G-YX0Z), the National Science Foundation of China (51379048) and the International S\&T Cooperation Program of China (S2014GAT013).

1. J.-A. E. Månson, M. D. Wakeman, and N. Bernet, "Composite processing and manufacturing - An overview," in: A. Kelly and C. Zweben (Eds.), Comprehensive Composite Materials, Vol. 2: Polymer Matrix Composites, Elsevier Science Ltd, Amsterdam, The Netherlands (2000), pp. 577-607.

2. M. Hou, L. Ye, and Y. W. Mai, "Manufacturing process and mechanical properties of thermoplastic composite components," J. Mater. Process. Technol., 63, 334-338 (1997).

3. Z. Y. Jiang, S. Siengchin, and L. M. Zhou, "Poly(butylene terephthalate)/silica nanocomposites prepared from cyclic butylene terephthalate," Composites: Part A, 40, 273-278 (2009).

4. K. Pang, R. Kotek, and A. Tonelli, "Review of conventional and novel polymerization processes for polyesters," Progr. Polym. Sci., 31, No. 11, 1009-1037 (2006).

5. J. Baets, M. Dutoit, J. Devaux, and I. Verpoest, "Toughening of glass fiber reinforced composites with a cyclic butylene terephthalate matrix by addition of polycaprolactone," Composites: Part A, 39, 13-18 (2008).

6. H. L. Chen, W. Yu, and C. X. Zhou, "Entropically-driven ring-opening polymerization of cyclic butylene terephthalate: Rheology and kinetics," Polym. Eng. Sci., 52, No. 1, 91-101 (2012).

7. P. Hodge and H. M. Colquhoun, "Recent word on entropically-driven ring-opening polymerizations: some potential applications," Polym. Adv. Technol., 16, No. 2, 84-94 (2005).

8. H. Parton, J. Baets, P. Lipnik, et al., "Properties of poly(butylene terephthatlate) polymerized from cyclic oligomers and its composites," Polymer, 46, 9871-9880 (2005).

9. P. Fabbri, E. Bassoli, S. B. Bon, and L. Valentini, "Preparation and characterization of poly(butylene terephthalate)/grapheme composites by in-situ polymerization of cyclic butylene terephthalate," Polymer, 53, No. 4, 897-902 (2012). 
10. I. Yamaoka, "Anisotropic behaviour of styrene-butadiene-styrene triblock copolymer/ methyl methacrylate-styrene copolymer blends," Polymer, 39, No. 5, 1007-1257 (1998).

11. S. Vivekanandhan, M. Misra, and A. K. Mohanty, "Thermal, mechanical, and morphological investigation of injection molded poly(trimethylene terephthalate)/ carbon fiber composites," Polym. Compos., 33, No. 11, 1933-1940 (2012).

12. G. Lanciano, A. Greco, A. Maffezzoli, and L. Mascia, "Effects of thermal history in the ring opening polymerization of CBT and its mixtures with montmorillonite on the crystallization of the resulting poly(butylene terephthalate)," Thermochim. Acta, 493, 61-67 (2009).

13. Z. A. M. Ishad, P. P. Shang, and J. Karger-Kocsis, "A modulated DSC study on the in situ polymerization of cyclic butylene terephthalate oligomers," J. Therm. Anal. Calorim., 84, No. 3, 637-641 (2006).

14. Z. Hashin, "Analysis of composite materials - A survey," J. Appl. Mech., 50, No. 3, 481-505 (1983).

15. S. B. Brahim and R. B. Cheikh, "Influence of fibre orientation and volume fraction on the tensile properties of unidirectional Alfa-polyester composite," Compos. Sci. Technol., 67, 140-147 (2007).

16. A. A. Abdulmajeed, T. O. Narhi, P. K. Vallittu, and L. V. Lassila, "The effect of high fiber fraction on some mechanical properties of unidirectional glass fiber-reinforced composite," Dent. Mater., 27, No. 4, 313-321 (2011).

Received 20. 10. 2014 